\title{
Probing level renormalization by sequential transport through double quantum dots
}

\author{
Bernhard Wunsch, ${ }^{1, *}$ Matthias Braun, ${ }^{2}$ Jürgen König, ${ }^{2}$ and Daniela Pfannkuche ${ }^{1}$ \\ ${ }^{1}$ I. Institut für Theoretische Physik, Universität Hamburg, Jungiusstr. 9, 20355 Hamburg, Germany \\ ${ }^{2}$ Institut für Theoretische Physik III, Ruhr-Universität Bochum, 44780 Bochum, Germany
}

(Dated: September 29, 2018)

\begin{abstract}
We study electron transport through double quantum dots in series. The tunnel coupling of the discrete dot levels to external leads causes a shift of their energy. This energy renormalization affects the transport characteristics even in the limit of weak dot-lead coupling, when sequential transport dominates. We propose an experimental setup which reveals the renormalization effects in either the current-voltage characteristics or in the stability diagram.
\end{abstract}

PACS numbers: 73.21.La, 73.23.Hk, 73.63.Kv

\section{INTRODUCTION}

Serial double quantum dots are ideal systems to investigate various quantum mechanical effects such as molecular binding ${ }^{1,2}$ or coherent dynamics ${ }^{3}$ between the constituent dots. Furthermore, they are considered as an implementation of a charge ${ }^{4}$ or spin qubit. ${ }^{5}$ Elaborate experimental techniques were developed to control and characterize double-dot structures ${ }^{6-8}$ and many information about the system can be deduced from the electric conductance through the device. ${ }^{9}$ Recent experiments include the measurements of quantum mechanical level repulsion due to interdot coupling ${ }^{10}$ as well as due to external magnetic fields, ${ }^{11}$ the detection of molecular states in a double dot dimer, ${ }^{12}$ and the observation of coherent time evolution of the dot states. ${ }^{3}$

Transport through serial double dots, as depicted in Fig. 1, inherently visualizes the basic quantum mechanical concept of coherent superposition of charge states. ${ }^{13}$ The states that are coupled to the left and right lead, the localized states in the left and right dot, respectively, are no energy eigenstates of the double dot. This leads to oscillations of the electron in the double dot as it was shown in recent experiments. ${ }^{3,4}$ To account for this internal dynamics, descriptions using classical rates only, are insufficient, which is why approaches including nondiagonal density matrix elements for the double dot have been developed. ${ }^{14-17,23}$.

In this paper, we propose to use a serial double quantum dot to probe another consequence of quantum mechanics: the energy level renormalization of the quantum dot levels due to tunnel coupling to a reservoir. This idea is based on two properties of a serial dot system. First, the left and right dot levels are tunnel-coupled to different reservoirs. Since the level renormalization is a function of the level energy, the lead chemical potential, and the tunnel coupling, the energy shift of the left and right dot levels is, in general, different. Second, the conductance through the double dot is very sensitive to the difference of the energy levels. It shows a resonant behavior with the width given by the tunnel couplings, ${ }^{18}$ which can be much smaller than the temperature. This sharpness of the resonance makes the conductance a valuable experi-

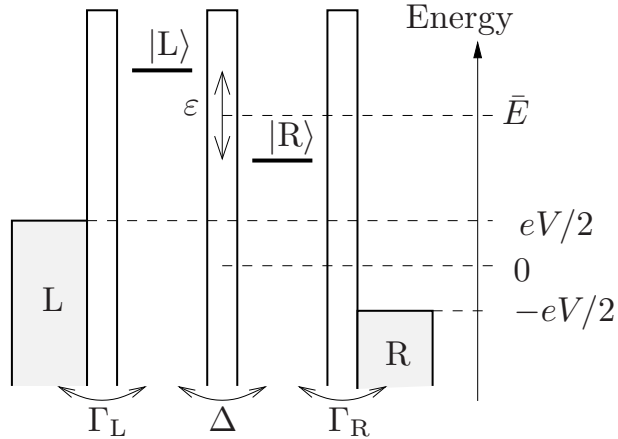

FIG. 1: Schematic energy profile for a double dot coupled in series to two reservoirs. Each reservoir is coupled to the dot of the corresponding side by the coupling strength $\Gamma_{r}$. The inter-dot coupling is determined by $\Delta$. The energies of the two dot states are characterized by the mean energy $\bar{E}$ and their relative distance $\varepsilon$.

mental tool, for example to measure the shell structure of quantum dots. ${ }^{19}$

It is well known ${ }^{20,21}$ that tunnel coupling to reservoirs renormalizes the energy levels. In single-dot geometries such an energy renormalization is only accessible in transport of higher order in the tunnel coupling strength. As we will show below, this is different for the serial double dot geometry, for which renormalization effects are visible in the conductance already in the limit of weak dot-lead coupling, described by transport to first order (sequential tunneling) in the tunnel-coupling strength $\Gamma=\Gamma_{\mathrm{L}}+\Gamma_{\mathrm{R}}$.

The paper is organized as follows: In Sec. II we present the model Hamiltonian for the double dot. ${ }^{15-17,23,23,24}$ In Sec. III we derive the stationary density matrix and the $d c$-current for arbitrary bias voltages. In Sec. IV, we discuss how renormalization effects appear in the $d c-$ current through the double dot. We draw our conclusions in Sec. V, followed by some technical notes in the appendices. In App. A we make explanatory notes of the diagrammatic technique, and in App. B we give an illustrative reformulation of the master equation in terms of a pseudo spin. 


\section{MODEL}

We consider a double quantum dot, contacted in series, which is described by the Hamiltonian ${ }^{23}$

$$
H=\sum_{r=\mathrm{L}, \mathrm{R}} H_{r}+H_{\mathrm{D}}+H_{\mathrm{T}} .
$$

The first part of the Hamiltonian describes the electric contacts on the left $(\mathrm{L})$ and right $(\mathrm{R})$ side. These contacts are modeled by large reservoirs of noninteracting electrons $H_{r}=\sum_{k, \sigma} \varepsilon_{r k} c_{r k \sigma}^{+} c_{r k \sigma}$. Here $c_{r k \sigma}, c_{r k \sigma}^{+}$denote the annihilation and creation operators for electrons in the reservoir $r \in\{\mathrm{L}, \mathrm{R}\}$ with spin $\sigma$. The reservoirs are assumed to be in equilibrium, so that they can be characterized by the Fermi distribution $f_{\mathrm{L} / \mathrm{R}}(\omega)$. An applied bias voltage $V$ is modeled by different chemical potentials in the left and right contact $f_{\mathrm{L} / \mathrm{R}}(\omega)=f(\omega \pm \mathrm{eV} / 2)$.

The second part of the Hamiltonian, $H_{\mathrm{D}}$, describes two dots, containing one electronic level each, which are coupled by the Coulomb interaction:

$$
H_{\mathrm{D}}=\sum_{r=\mathrm{L}, \mathrm{R}} E_{r} n_{r}+U n_{\mathrm{L}} n_{\mathrm{R}}+U^{\prime}\left(n_{\mathrm{L} \uparrow} n_{\mathrm{L} \downarrow}+n_{\mathrm{R} \uparrow} n_{\mathrm{R} \downarrow}\right) .
$$

Here, $n_{i \sigma}=d_{i \sigma}^{+} d_{i \sigma}$ and $n_{i}=\sum_{\sigma} d_{i \sigma}^{+} d_{i \sigma}$ are the occupation number operators for $\operatorname{dot} i \in\{\mathrm{L}, \mathrm{R}\}$ with spin $\sigma$, where $d_{i \sigma}, d_{i \sigma}^{+}$being the annihilation and creation operators of an electron on dot $i$ with spin $\sigma$. Each dot consists of a single electronic level at the energy $E_{\mathrm{L} / \mathrm{R}}$ measured relative to the equilibrium chemical potential of the leads. We parameterize the levels by their average energy $\bar{E}=\left(E_{\mathrm{L}}+E_{\mathrm{R}}\right) / 2$ and their difference $\varepsilon=E_{\mathrm{L}}-E_{\mathrm{R}}$, so that $E_{\mathrm{L} / \mathrm{R}}=\bar{E} \pm \varepsilon / 2$. Double occupation of one individual dot is associated with the intradot charging energy $U^{\prime}$. Simultaneous occupation of the both dots with one electron each costs the interdot charging energy $U$ with $U^{\prime} \gg U$. States with three or more electrons in the double dot are not considered in the following. The remaining eigenstates of $H_{\mathrm{D}}$, then, are: both dots empty $|0\rangle$, one electron with spin $\sigma$ in the left $|\mathrm{L} \sigma\rangle$ or right dot $|\mathrm{R} \sigma\rangle$, and one electron in each dot $\left|\mathrm{L} \sigma \mathrm{R} \sigma^{\prime}\right\rangle$. We assume that the intra-dot charging energy always exceeds the lead Fermi energies. Therefore the states with two electrons in the same dot $|\mathrm{L} \sigma \mathrm{L} \bar{\sigma}\rangle$ and $|\operatorname{R} \sigma \mathrm{R} \bar{\sigma}\rangle$ will have a vanishing occupation probability. However, these states will appear as intermediate (virtual) states in our calculation, providing a natural high-energy cut-off.

The third part $H_{\mathrm{T}}=H_{\Delta}+H_{\Gamma}$ of the Hamiltonian Eq. (1) describes both, tunneling between the two dots, $H_{\Delta}$, as well as tunneling between dots and leads, $H_{\Gamma}$,

$$
\begin{aligned}
& H_{\Delta}=-\frac{\Delta}{2} \sum_{\sigma}\left(d_{\mathrm{L} \sigma}^{+} d_{\mathrm{R} \sigma}+d_{\mathrm{R} \sigma}^{+} d_{\mathrm{L} \sigma}\right) \\
& H_{\Gamma}=\sum_{k \sigma} t_{\mathrm{L} k} c_{\mathrm{L} k \sigma}^{+} d_{\mathrm{L} \sigma}+t_{\mathrm{R} k} c_{\mathrm{R} k \sigma}^{+} d_{\mathrm{R} \sigma}+\text { h.c. }
\end{aligned}
$$

Due to the serial geometry, an electron from the right (left) reservoir can only tunnel to the right (left) dot.
The tunnel coupling of reservoir $r$ to the corresponding dot is characterized by the coupling strength $\Gamma_{r}(\omega)=$ $2 \pi \sum_{k}\left|t_{r k}\right|^{2} \delta\left(\varepsilon_{r k}-\omega\right)$. We consider only spin conserving tunneling processes, and assume flat bands in the reservoirs, which yields energy independent couplings $\Gamma_{r}$. Furthermore, we choose the interdot tunnel coupling amplitude $\Delta$ as a positive, real parameter, which can be always achieved by a proper gauge transformation.

\section{KINETIC EQUATION}

In the following section we calculate the stationary reduced density matrix $\rho_{\text {st }}$ for the double dot system and the $d c$-current through the system. The reduced density matrix of the double-dot is obtained from the density matrix of the whole system by integrating out the reservoir degrees of freedom. The Liouville equation for the reduced density matrix then has the following structure:

$$
0=i \hbar \frac{d}{d t} \boldsymbol{\rho}_{\mathrm{st}}=\left[H_{\mathrm{D}}, \boldsymbol{\rho}_{\mathrm{st}}\right]+\left[H_{\Delta}, \boldsymbol{\rho}_{\mathrm{st}}\right]+\hat{\Sigma} \boldsymbol{\rho}_{\mathrm{st}} .
$$

The first two parts represent the internal dynamics on the double dot, which depends on the level separation $\varepsilon$ and the interdot coupling $\Delta$. The third part of Eq. (5) accounts for the tunnel coupling between double dot and external reservoirs. The fourth order tensor $\hat{\Sigma}$ contains imaginary and real parts, associated with particle transfer processes and with tunnel induced energy renormalization of the dot levels, respectively. The latter has been neglected in previous works. ${ }^{16,17,23}$ We calculate $\hat{\Sigma}$ using a real-time diagrammatic approach ${ }^{21,25}$ as explained in App. A. Also alternative methods are available such as Bloch-Redfield theory. ${ }^{22,26}$

In the following we concentrate on the limit of weak tunnel coupling between double dot and leads. Therefore, we calculate $\hat{\Sigma}$ to lowest order in the tunnel-coupling strength $\Gamma=\Gamma_{\mathrm{L}}+\Gamma_{\mathrm{R}}$, which defines the so-called sequential-tunneling approximation. This approximation implies that all tunneling events are independent from each other, which is fulfilled for $k_{\mathrm{B}} T \gg \Gamma$. Since the correlations generated in the bath during a tunnel process decay on the time scale $\hbar / k_{\mathrm{B}} T,{ }^{27}$ (this follows from the dependence of the tunneling line in Fig. 4-6 on its extension in time), while the average time between consecutive tunneling events is given by the inverse of the coupling strength $\hbar / \Gamma$, higher order, coherent tunneling events are suppressed by the condition $k_{\mathrm{B}} T \gg \Gamma$ and may be neglected.

The energy eigenstates of the double dot subsystem $H_{\mathrm{D}}+H_{\Delta}$ are the bonding and anti-bonding states with energies $E_{\mathrm{b} / \mathrm{a}}=\bar{E} \mp \Delta_{\mathrm{ab}}$ where $\Delta_{\mathrm{ab}}=\sqrt{\Delta^{2}+\varepsilon^{2}}$ denotes their energy splitting. This identifies $\Delta_{\mathrm{ab}}$ as frequency of the charge oscillations, ${ }^{3,4}$ and $\Delta$ as minimum distance between the bonding and anti-bonding eigenstates as function of the left and right energy level. ${ }^{10}$

If the splitting exceeds the intrinsic broadening of the levels, $\Delta_{\mathrm{ab}} \gg \Gamma$, then the internal oscillations are fast, 
and transport through the double-dot system takes place through two separate incoherent levels. In this case, offdiagonal matrix elements of the stationary density matrix vanish, which can be seen from the expansion of the Liouville equation $0=i \hbar \frac{d}{d t} P_{\mathrm{b}}^{\mathrm{a}}=\Delta_{\mathrm{ab}} P_{\mathrm{b}}^{\mathrm{a}}+O(\Gamma)$, where $P_{\chi_{2}}^{\chi_{1}}$ denotes the matrix element $P_{\chi_{2}}^{\chi_{1}}=\left\langle\chi_{1}\left|\rho_{\text {st }}\right| \chi_{2}\right\rangle$ of the reduced density.

The more interesting transport regime is in the opposite limit, $\Delta_{\mathrm{ab}} \lesssim \Gamma$, where the external coupling strongly modifies the internal dynamics, which is captured by the off-diagonal elements of the reduced density matrix. ${ }^{17,23}$ Combined with the validity condition for sequential tunneling, i.e. $\Gamma \ll k_{\mathrm{B}} T$ this implies $\Delta_{\mathrm{ab}} \ll k_{\mathrm{B}} T$, i.e., internal oscillations are slow as compared to the time scale for the correlations during a tunneling event. As a consequence, the localized states $|\mathrm{L} \sigma\rangle$ and $|\mathrm{R} \sigma\rangle$ can be used as eigenstates of the double dot in the calculation of $\hat{\Sigma}$, which facilitates the interpretation of the dynamics. Technically, the condition $\Delta_{\mathrm{ab}} \lesssim \Gamma \ll k_{\mathrm{B}} T$ means that for a consistent theory, we do not only expand $\hat{\Sigma}$ in Eq. (5) to first order in $\Gamma$, but also have to expand it to zeroth order in $\Delta_{\mathrm{ab}}$. This is accomplished by replacing the energies $E_{\mathrm{L} / \mathrm{R}}$ arising in the calculation of $\hat{\Sigma}$ by the mean level energy $\bar{E}=\left(E_{\mathrm{L}}+E_{\mathrm{R}}\right) / 2$. (Therefore our formulas only contain the Fermi functions at the average single particle level $f_{r}(\bar{E})$, while energies of the order of the interdot tunneling or the level separation are smeared by temperature). It is worth to point out, that by using the localized states as basis of the $\hat{\Sigma}$ (i.e. by calculating the transition rates in the localized basis) one is automatically limited to the regime $\Delta, \varepsilon \ll k_{\mathrm{B}} T$. ${ }^{14,16,17,23}$

The technical details of how to calculate $\hat{\Sigma}$ are described in App. A. The master Eq. (5) then must be solved under the constraint of probability normalization $\operatorname{Tr}\left[\boldsymbol{\rho}_{\text {st }}\right]=1$. The stationary current $I$ is given by the time derivative of the expectation value of the total number of electrons in either the left or the right lead. For the lowest-order expansion used in the present context, the current can alternatively be written in the form ${ }^{23}$

$$
I=-e \frac{i}{\hbar}\left\langle\left[H_{\Delta}, n_{\mathrm{L}}\right]\right\rangle=-\frac{e}{\hbar} \Delta \operatorname{Im}\left(\sum_{\sigma} P_{\mathrm{R} \sigma}^{\mathrm{L} \sigma}\right)
$$

where Im denotes the imaginary part. In App. B we give an analytical solution for the current as function of bias voltage and gate voltages. In the following we discuss our results.

Instead of working with an off-diagonal density matrix one may switch to a pseudo spin representation of the problem. Then this double-dot transport problem shows similarities to the system of a quantum dot connected to ferromagnetic leads. ${ }^{28,29}$ This will be discussed in App. B.

\section{DISCUSSION}

The stationary current takes the form

$$
I=\frac{e}{\hbar} \Delta^{2} \frac{A}{B^{2}+\varepsilon_{\text {ren }}^{2}}
$$

The numerical factors $A$ and $B$ (the explicit form is given in App. B) depend only on the tunnel coupling constants $\Gamma_{\mathrm{L}}, \Gamma_{\mathrm{R}}$, and $\Delta$ as well as on the Fermi distribution functions $f_{\mathrm{L} / \mathrm{R}}(\bar{E})$ and $f_{\mathrm{L} / \mathrm{R}}(\bar{E}+U)$ of the left and right lead, but not on the level energy difference $\varepsilon$. The current in Eq. (7) shows the well-known ${ }^{18,23}$ Lorentzian dependence on the energy separation $\varepsilon_{\text {ren }}$ between left and right dot level. However the energy separation is affected by the renormalization of the bare localized levels:

$$
\varepsilon_{\text {ren }}=\varepsilon+\Delta E_{\mathrm{L}}-\Delta E_{\mathrm{R}}
$$

This is the central statement of the present paper. The energy shift $\Delta E_{r}$ of the energy level in dot $r$, caused by the external tunnel coupling, is given by

$$
\Delta E_{r}=\phi_{r}(\bar{E})-2 \phi_{r}(\bar{E}+U)+\phi_{r}\left(\bar{E}+U^{\prime}\right)
$$

with

$$
\phi_{r}(\omega)=\frac{\Gamma_{r}}{2 \pi} \operatorname{Re} \Psi\left(\frac{1}{2}+i \beta \frac{\omega-\mu_{r}}{2 \pi}\right) .
$$

Here, Re denotes the real part, $\Psi$ is the digamma function, and $\mu_{\mathrm{L} / \mathrm{R}}= \pm e V / 2$ the leads' chemical potentials. We want to emphasize that this energy renormalization is not due to a capacitative but rather due to the tunnel coupling to the reservoirs. Furthermore, it vanishes in the noninteracting case $U=U^{\prime}=0$. The intradot charging energy $U^{\prime}$ (which we usually treat as infinite to avoid double occupation of one dot) serves as a natural cut off for the energy renormalization in Eq. (9). This is the reason why we allowed the intermediate states $\chi_{5}$ in App. A to occupy these states.

The energy shift of the localized levels is proportional to the tunnel coupling strength and depends on the dot level positions relative to the Fermi energy. The renormalized level separation as function of the bias voltage is plotted in Fig. 2a. The renormalized level separation $\varepsilon_{\text {ren }}$ reaches a (local) extremum each time, when the Fermi energy of a lead becomes resonant with the energy needed for single $\left(\mu_{r}=\bar{E}\right)$ or double occupation $\left(\mu_{r}=\bar{E}+U\right)$.

Fig. $2 \mathrm{~b}$ shows the current as function of the transport voltage taking the level shift into account (solid line). By neglecting the level shifts (dashed line in Fig. 2b), the current shows a typical Coulomb staircase. The steps occur when a lead chemical potential aligns with an electronic level in the double dot. Since the bare energy level separation $\varepsilon$ as well as the interdot tunneling $\Delta$ shall be of the order of or smaller $\Gamma \geq\{\Delta, \varepsilon\}$ and we consider $\Gamma<k_{\mathrm{B}} T$, the different single particle states are not resolved as individual steps in the $I-V$ staircase. The 


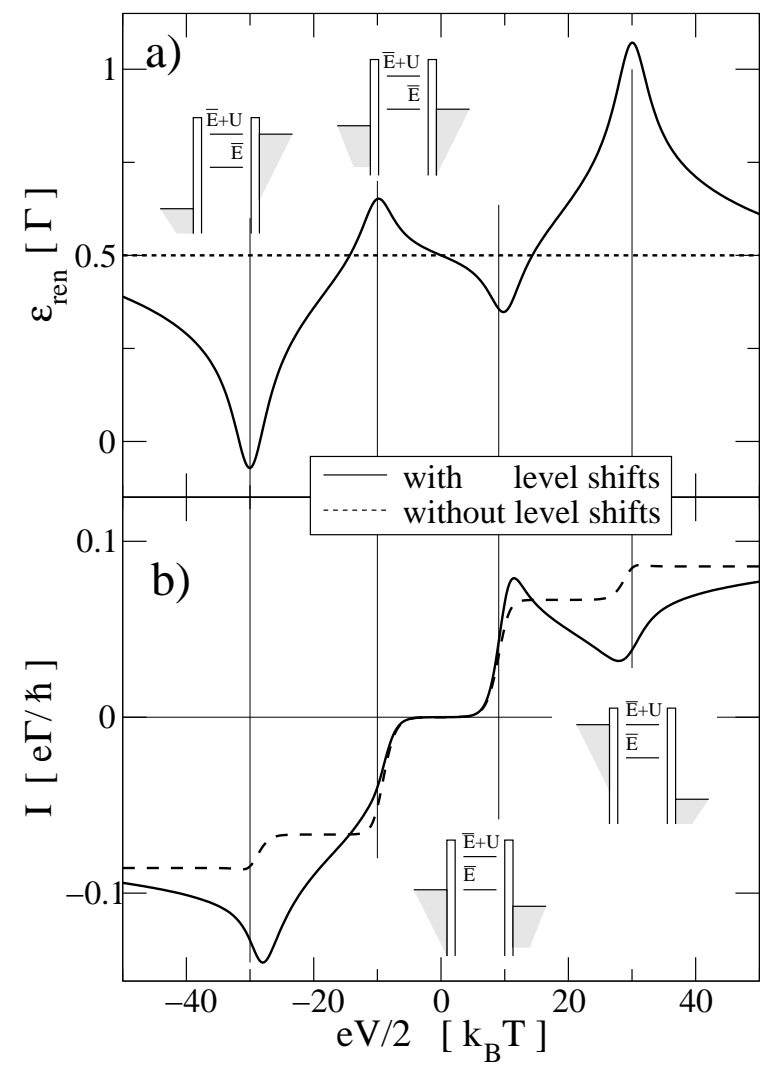

FIG. 2: Upper Part: Renormalized level spacing $\varepsilon_{\text {ren }}$ (solid line) between the electronic levels in the left and right dot as function of the transport voltage $V . \varepsilon_{\text {ren }}$ is extremal, when the chemical potential of a lead aligns with the energy needed for either single $(\bar{E})$ or double occupation $(\bar{E}+U)$. Lower part: Current-voltage characteristics for bare (dashed line) and renormalized level spacing (solid line). Renormalization of energy levels leads to an asymmetric current-voltage characteristic. The current increases (decreases) whenever the level spacing is reduced (increased) with respect to the bare value. Plot parameters are: $\varepsilon=\Delta=\Gamma_{\mathrm{R}}=\Gamma_{\mathrm{L}}=\Gamma / 2$, $\bar{E}=10 k_{\mathrm{B}} T, U=20 k_{\mathrm{B}} T$, and $U^{\prime}=100 k_{\mathrm{B}} T$.

tunnel induced renormalization leads to additional features on the staircase (solid line in Fig. 2b). Whenever the magnitude of the renormalized level spacing grows (drops) the current decreases (increases). This leads to a suppression or an enhancement of the current around the steps of the $I-V$ characteristic, leading to regions of negative differential conductance. The width of these feature is of the order of the charging energy and can exceed temperature and coupling strength significantly.

Neglecting renormalization effects and assuming symmetric coupling to the reservoirs $\left(\Gamma_{L}=\Gamma_{R}\right)$, the current through the double dot is an odd function of the transport voltage (see dashed line in Fig. 2b). This is no longer the case when renormalization is taken into account (see solid line in Fig. 2b). The reason for this asymmetry is that even though the change of asymmetry, $\Delta E_{\mathrm{L}}-\Delta E_{\mathrm{R}}$, caused by level renormalization is antisymmetric with re- spect to the bias voltage, this in not true for the total asymmetry $\varepsilon_{r e n}=\varepsilon+\Delta E_{\mathrm{L}}-\Delta E_{\mathrm{R}}$ due to the nonvanishing bare splitting $\varepsilon$ (see Fig. 2a). A comparable asymmetry in transport through two coupled dots was recently observed by Ishibashi et al. ${ }^{30}$ and theoretically described by Fransson et al. ${ }^{20}$ However, a negative differential conductance feature can not be uniquely linked to such renormalization effects. Due to interface capacities the level positions in the left and right dot are always affected by the transport voltage in real experiments. ${ }^{18,31}$

To exclude the effect of interface capacities, we propose a different experiment: measuring the current $I\left(E_{\mathrm{L}}, E_{\mathrm{R}}\right)$ at a constant transport voltage as function of the left and right gate voltages on the dots. The resulting stability diagram is plotted in Fig. 3a. Elastic sequential tunneling from the left to the right dot is possible if $E_{\mathrm{L}} \approx E_{\mathrm{R}}$. Furthermore electron transport from the left to the right reservoir takes only place if the dot level for single $(\bar{E})$ or double occupation $(\bar{E}+U)$ is located in the bias voltage window. Therefore the current resonance forms two stripes in the regions $-e V / 2<\bar{E}<e V / 2$ and $-U-e V / 2<\bar{E}<-U+e V / 2$. Away from the current stripes the occupation number of the left and right dot $\left(N_{\mathrm{L}}, N_{\mathrm{R}}\right)$ is fixed, and no current can cross the structure. For a detailed discussion on stability diagrams for transport through double dots we refer to the review of van der Wiel. et al. ${ }^{6}$

In the absence of renormalization effects, the current stripes would exactly coincide with the condition $E_{\mathrm{L}}=E_{\mathrm{R}}$. By plotting the current as function of the mean level position $\bar{E}=\left(E_{\mathrm{L}}+E_{\mathrm{R}}\right) / 2$ and the relative energy difference $\varepsilon=E_{\mathrm{L}}-E_{\mathrm{R}}$, one would therefore expect a straight horizontal line. Instead, the maximum of the current follows the renormalization shift, where the condition $\varepsilon_{\text {ren }}=0$ is fulfilled, see Fig. $3 \mathrm{~b}$. The shift of the resonance is of order $\Gamma$ as shown in Eq. (9) and can be small on the scale of bias voltage or temperature. The width of the current maxima in the stability diagram in Fig. 3 is not determined by temperature but rather by the dominant coupling strength ${ }^{18} \max (\Gamma, \Delta)$. Therefore the resonance width is sharp enough to be able to measure the renormalization of energy levels if $\Gamma \gtrsim \Delta$ as used in Fig. 3.

In the nonlinear transport regime $\Delta E_{r}$ depends on $\bar{E}$ and therefore the current stripes in Fig. 3 are bent and tilted against each other. This dependence can be used as a stringent experimental prove of the renormalization of energy levels. Due to internal cross capacities, always appearing in real experiments, the gate voltage of one dot is a linear function of the gate voltage of the other dot. Therefore the stability diagram $I\left(V_{\mathrm{L}}, V_{\mathrm{R}}\right)$ as plotted in Fig. 3a would experience a linear shear transformation. However straight (parallel) lines stay straight (parallel). Thus, cross capacities can not mimic the bending due to renormalization effects.

In real experiments in addition to the resonant current stripes explained here, further features can arise due to inelastic processes, cotunneling, or due to excited levels 

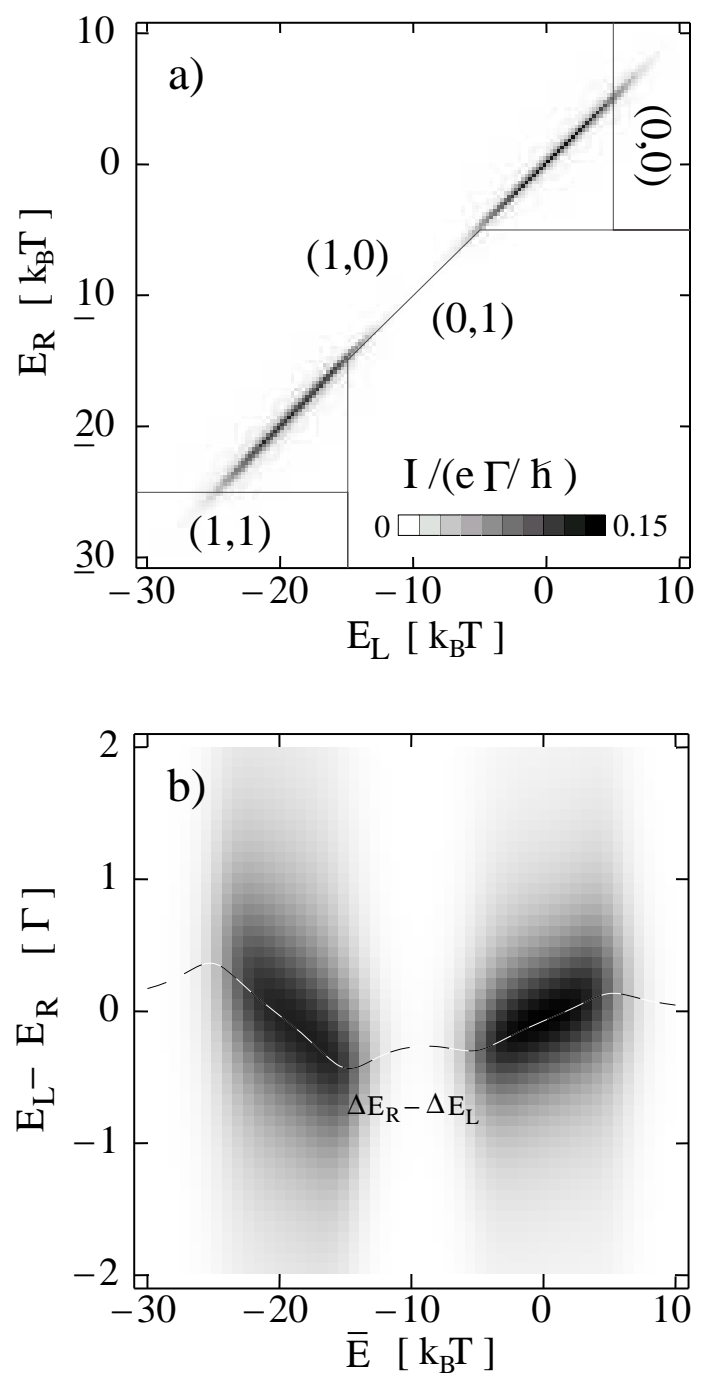

FIG. 3: Upper panel: Stability diagram $I\left(E_{\mathrm{L}}, E_{\mathrm{R}}\right)$ of the current through the double dot in the nonlinear transport regime. Well inside the areas, separated by the black line, the occupation of the individual dots is fixed to the written values $\left(N_{\mathrm{L}}, N_{\mathrm{R}}\right)$. Elastic sequential current can cross the structure for $E_{\mathrm{L}} \approx E_{\mathrm{R}}$ and either $-e V / 2<\bar{E}<e V / 2$ or $-e V / 2<$ $\bar{E}+U<e V / 2$ resulting in two current stripes. Lower panel: Gray scale plot of the current as function of the average single particle energy $\bar{E}$ and the bare level separation $\varepsilon=E_{\mathrm{L}}-E_{\mathrm{R}}$. The different renormalization of left and right level shifts the current maxima by $\Delta E_{\mathrm{R}}-\Delta E_{\mathrm{L}}$ (dashed black-white line) where $\varepsilon_{\text {ren }}=0$. This leads to a tilting of the current stripes relative to each other. Relevant plot parameters are $k_{\mathrm{B}} T=$ $2 \Gamma, \Gamma_{\mathrm{L}}=\Gamma_{\mathrm{R}}=\Delta=\Gamma / 2, V=10 k_{\mathrm{B}} T, U^{\prime}=100 k_{\mathrm{B}} T$, and interdot charging energy $U=20 k_{\mathrm{B}} T$.

within the bias voltage window. ${ }^{6}$ These effects mainly lead to features within the triangles below the current strips in Fig. 3a and are expected not to interfere with our presented results.

Finally we compare our result obtained for the stationary current Eq. (B7) with previous theoretical works. For this we set the Fermi functions to $f_{\mathrm{L}}(\bar{E})=1$ and
$f_{\mathrm{L}}(\bar{E}+U)=f_{\mathrm{R}}(\bar{E})=f_{\mathrm{R}}(\bar{E}+U)=0$. This simplifies the current to:

$$
I=\frac{e}{\hbar} \frac{\Gamma_{\mathrm{R}} \Delta^{2}}{\Delta^{2}\left(2+\frac{\Gamma_{\mathrm{R}}}{2 \Gamma_{\mathrm{L}}}\right)+4\left(\varepsilon_{\mathrm{ren}}\right)^{2}+\Gamma_{\mathrm{R}}^{2}},
$$

Neglecting renormalization effects (setting $\varepsilon_{\text {ren }}=\varepsilon$ ), this equation reproduces Eq. (4.19) in the paper by Gurvitz, ${ }^{17}$. Choosing the voltages such that the dot structure can also be doubly occupied, i.e. $f_{\mathrm{L}}(\bar{E}+U)=$ $f_{\mathrm{L}}(\bar{E})=1$ and $0=f_{\mathrm{R}}(\bar{E})=f_{\mathrm{R}}(\bar{E}+U)$ one obtains Eq. (4.18) of Ref. 17.

Several publications assume, that if the lead Fermi energies are far away from the electronic states of the dots, then the principal value integrals (Eq. B5), leading to the renormalization, can be neglected. However the energy shifts are relevant on an energy scale given by the charging energy $U$, as shown in Fig. 2a. Therefore the assumption, that one can neglect renormalization effects and still exclude states with more than one electron occupying the double dot is not justified.

\section{CONCLUSIONS}

If a quantum dot is connected to a reservoirs, the tunnel coupling causes an energy renormalization of the electronic states. We derived the conductance of a double dot connected in series to external reservoirs for general bias voltages and temperatures, taking into account these energy renormalizations. We have shown, that the conductance of such a double dot structure is affected by the energy level shifts already in a lowest order expansion in the tunnel coupling strength, due to its high sensitivity on the relative detuning of energy levels. Therefore we propose to use a double-dot system as detector for these energy renormalization effects.

We present experimental consequences of the renormalization in the current-voltage characteristics and in the stability diagram for the double dot in the nonlinear transport regime. In the current-voltage characteristics we find prominent negative differential conductances in voltage windows of the order of the charging energy.

In the stability diagram of the double dot, we found that the current stripes arising as function of the gate voltages for left and right dot are tilted against each other and do not lie on a straight line, as it is the case when energy renormalization is neglected. We showed that the tilting of the current stripes is resolvable even in the sequential tunneling regime (i.e. for $\Gamma>k_{\mathrm{B}} T$ ) as long as the interdot tunneling, $\Delta$ is of the same order or smaller than the external coupling $\Gamma \geq \Delta$.

\section{Acknowledgments}

We thank S. Debald and B. Kubala, for fruitful discussions. This work was supported by the 
Deutsche Forschungsgemeinschaft via SFB 508 and under the Emmy-Noether program, through SFB 491 and GRK 726.

\section{APPENDIX A: DIAGRAMMATIC RULES}

With the definition $P_{\chi_{2}}^{\chi_{1}}:=\left\langle\chi_{1}\left|\rho_{\text {st }}\right| \chi_{2}\right\rangle$, the master equation Eq. (5) can be written as:

$$
\begin{aligned}
0=i \hbar \frac{d}{d t} P_{\chi_{2}}^{\chi_{1}}= & \left\langle\chi_{1}\left|\left[H_{\mathrm{D}}, \rho_{\mathrm{st}}\right]\right| \chi_{2}\right\rangle+\left\langle\chi_{1}\left|\left[H_{\Delta}, \rho_{\mathrm{st}}\right]\right| \chi_{2}\right\rangle \\
& +\sum_{\chi_{3}, \chi_{4}} \Sigma_{\chi_{2} \chi_{4}}^{\chi_{1} \chi_{3}} P_{\chi_{4}}^{\chi_{3}} .
\end{aligned}
$$

In the following we show, how we calculate the tensor $\Sigma_{\chi_{4} \chi_{2}}^{\chi_{3} \chi_{1}}$, where $\chi_{i} \in\left\{|0\rangle,|\mathrm{L} \sigma\rangle,|\mathrm{R} \sigma\rangle,\left|\mathrm{L} \sigma \mathrm{R} \sigma^{\prime}\right\rangle\right\}$ are the localized eigenstates of $H_{\mathrm{D}}$, including the spin degree of freedom.

We apply a diagrammatic technique, where each class of tunneling processes can be represented by a diagram. Its general derivation can be found in Ref. 21. Recently this technique was applied to Anderson-like Hamiltonians to investigate spin-valve effects, ${ }^{28}$ or signatures of the excitation spectrum in the Coulomb blockade. ${ }^{32}$

Within this approach, the tensor $\Sigma_{\chi_{4} \chi_{2}}^{\chi_{3} \chi_{1}}$ is represented as block diagram, which is a part of the Keldysh time contour as shown in Fig. 4. The upper and lower line of the Keldysh time contour $t_{\mathrm{K}}$ represent the propagation of the double dot system forward and backward in time. They connect the matrix element characterized by the labels on the left side with the matrix element characterized by the labels on the right side. In the sequential tunneling approximation all transitions are allowed where a single electron first leaves and then reenters the double dot or vice versa. The two tunnel Hamiltonians are represented by vertices on the propagators. These vertices are connected by the contraction of the lead Fermi operators (indicated by a dashed line). Each line is characterized by its energy $\omega$, the spin $\sigma$ of the transfered electron, as well as the corresponding reservoir label $r \in\{L, R\}$. A vertex with an outgoing (incoming) tunneling line represents an electron leaving (entering) the double dot on the specified side $r$. All possible transitions in lowest order in the external coupling $\Gamma$ belong to one of the eight diagrams depicted in Fig. 5.

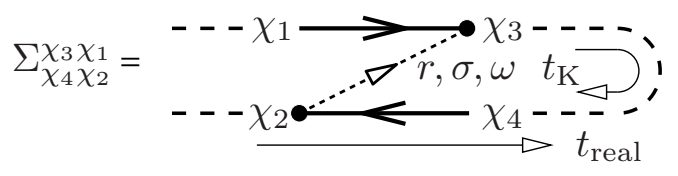

FIG. 4: Sketch of the structure of a diagram. The upper (lower) horizontal line denotes the forward (backward) propagator of the double dot system. The Keldysh time contour is labeled by $t_{\mathrm{K}}$, while the real time runs from left to right.

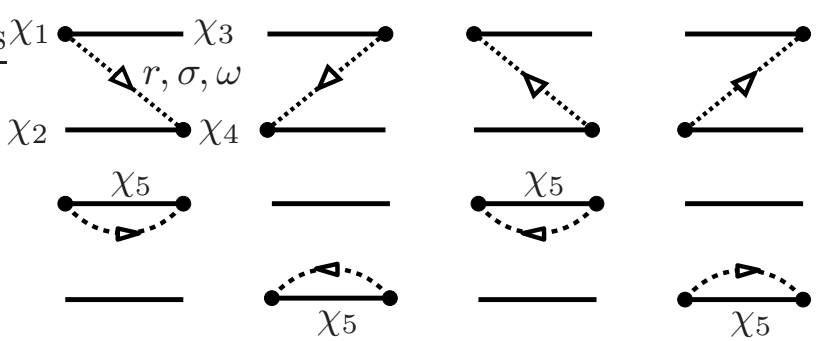

FIG. 5: All topologically different diagrams contributing to the tensor $\Sigma_{\chi_{4} \chi_{2}}^{\chi_{3} \chi_{1}}$ calculated in first order in the external coupling $\Gamma$. Labeling of the eigenstates at the four corners and of the tunneling line like in first diagram. $\chi_{5}$ labels an intermediate charge state of the double dot.

$\Sigma_{\chi_{4} \chi_{2}}^{\chi_{3} \chi_{1}}$ is given by the sum of all diagrams with the corresponding eigenstates at the four corners, see Fig. 5. The number of relevant diagrams is limited by spin and particle number conservation as well as to the serial system geometry. The rules to evaluate these diagrams in lowest order are:

1. Draw the upper and lower time contour. Add two tunnel vertices in any topological different way. The relevant criteria are the upper and lower contour, and the time ordering of the vertices on the real axes, not only on the Kelysh time contour. Assign to each free segment of the contour a state of the double dot and the corresponding energy. For 'bubble' diagrams like in the lower row of Fig. 5, an intermediate state $\chi_{5}$ participates.

2. The two vertices are connected by a tunnel line. Each tunnel line is labeled with the energy of the tunneling electron $\omega$, its reservoir label $r$ and its spin $\sigma$. Spin and reservoir label of the tunneling electron are uniquely determined by the eigenstates involved in the tunneling processes.

3. Assign to each diagram the resolvent $1 /\left(\Delta E+i 0^{+}\right)$ where $\Delta E$ is the difference between energies belonging to left going lines and energies belonging to right going lines (the tunneling line as well as the propagators).

4. The tunneling line connecting two vertices and labeled by the reservoir index $r$ gives rise to the factor

$$
\gamma_{r}^{ \pm}(\omega)=\frac{1}{2 \pi} \Gamma_{r} f_{r}^{ \pm}(\omega)
$$

Here, the Fermi function $f_{r}^{+}(\omega)=f_{r}(\omega)=1 /(1+$ $\left.\exp \left[\left(\omega-\mu_{r}\right) / k_{\mathrm{B}} T\right]\right)$ corresponds to a tunneling line that is backward directed in the Keldysh time ordering (compare Fig. 4), and $f_{r}^{-}(\omega)=1-f_{r}(\omega)$ corresponds to a tunneling line forward directed in the Keldysh time ordering.

5. Each diagram gets a prefactor $(-1)^{v}$ where $v$ is the number of vertices on the backward propagator. 
(This leads to an $(-1)$ for the diagrams in the upper row of Fig. 5.)

6. Sum over possible internal eigenstates $\chi_{5}$ and integrate over the energy $\omega$ of the tunneling electron.
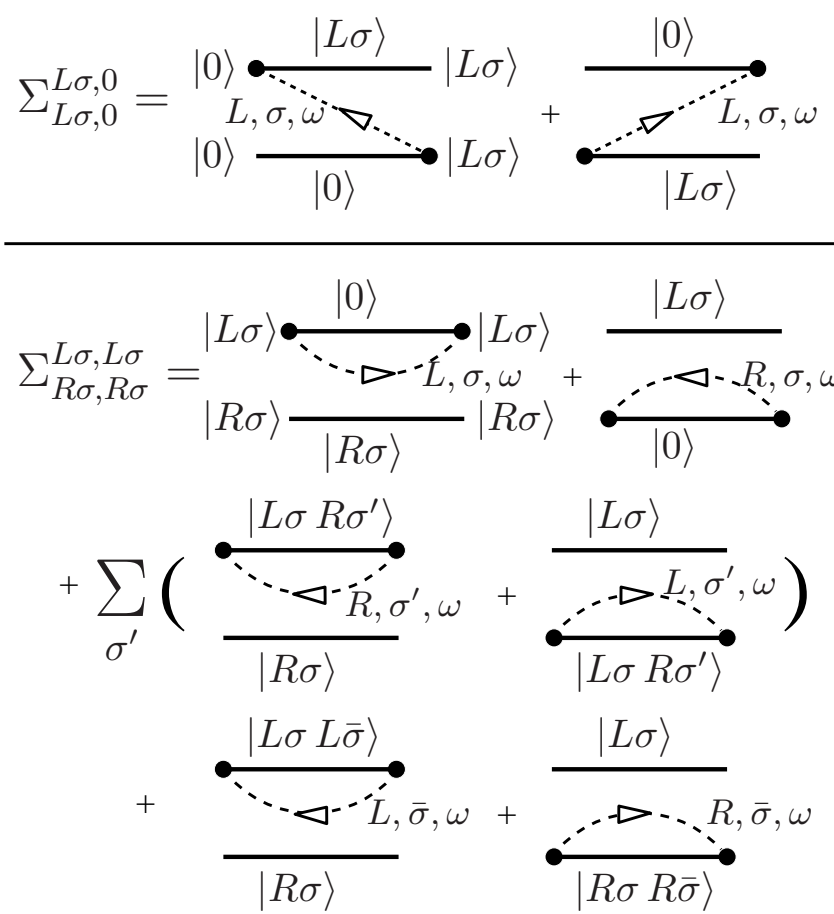

FIG. 6: Relevant diagrams contributing to two specific entries of $\hat{\Sigma}$, in a lowest order expansion in $\Gamma$. Every diagram corresponding to a specific entry is labeled by the same eigenstates at its four corners.

In the parameter regime we are interested in, the following relations hold: $k T>\Gamma \geq \varepsilon, \Delta$. Therefore the energy difference between the single particle states is not resolved by the Fermi functions in the reservoir, so that we have to approximate the eigenenergies of $\{|0\rangle,|\mathrm{L} \sigma\rangle$, $\left.|\mathrm{R} \sigma\rangle,\left|\operatorname{L} \sigma \mathrm{R} \sigma^{\prime}\right\rangle\right\}$ by $\left\{0, E_{\mathrm{L}} \approx E_{\mathrm{R}} \approx \bar{E}, 2 \bar{E}+U\right\}$. While we exclude a double occupation of a single dot for the initial or final states by setting $f_{r}\left(\bar{E}+U^{\prime}\right)=0$ we allow the intermediate state $\chi_{5}$ to be in such a state. These states have the eigenenergy $2 \bar{E}+U^{\prime}$.

In Fig. 6, we show as examples the diagrammatic expansion of the tensor elements $\Sigma_{\mathrm{L} \sigma, 0}^{\mathrm{L} \sigma, 0}$ and $\Sigma_{\mathrm{R} \sigma, \mathrm{R} \sigma}^{\mathrm{L} \sigma \mathrm{L} \sigma} \cdot \Sigma_{\mathrm{L} \sigma, 0}^{\mathrm{L} \sigma, 0}$ is purely imaginary and its magnitude has the meaning of a transition rate for a tunneling-in process starting from the empty double dot and resulting in a single electron with spin $\sigma$ sitting in the left dot. In contrast, $\Sigma_{\mathrm{R} \sigma, \mathrm{R} \sigma}^{\mathrm{L} \sigma}$ also has a real part which renormalizes the energy levels. Calculated in lowest order in $\Gamma$, each element of the tensor $\hat{\Sigma}$ can be expressed by terms of the form:

$$
X_{r}^{(n, m)}(E)=\int d \omega \frac{\gamma_{r}^{n}(\omega)}{m(E-\omega)+i 0^{+}},
$$

where $n$ and $m$ are either $(-)$ or $(+)$. In this notation, the algebraic expression for $\Sigma_{\mathrm{R} \sigma, \mathrm{R} \sigma}^{\mathrm{L} \sigma, \mathrm{L} \sigma}$ is:

$$
\begin{aligned}
\Sigma_{\mathrm{R} \sigma \mathrm{R} \sigma}^{\mathrm{L} \sigma \mathrm{L} \sigma}= & X_{r}^{(-,+)}(\bar{E})+X_{r}^{(-,-)}(\bar{E}) \\
& +g_{\sigma}\left(X_{r}^{(+,+)}(\bar{E}+U)+X_{r}^{(+,-)}(\bar{E}+U)\right) \\
& +\left(g_{\sigma}-1\right)\left(X_{r}^{(+,+)}\left(\bar{E}+U^{\prime}\right)+X_{r}^{(+,-)}\left(\bar{E}+U^{\prime}\right)\right)
\end{aligned}
$$

where, within this appendix, we allow for an arbitrary spin degeneracy $g_{\sigma}$. Since $f_{r}\left(\bar{E}+U^{\prime}\right)=0$ the imaginary part of the last row vanishes, however this is not the case for the real part, which causes the level renormalization. The real part of the diagrams is determined by the principal values of the integrals in Eq. (A2) and can be expressed as a sum over digamma functions, see Eq. (9).

Since the Hamiltonian given in Eq. (1) is independent of the orientation of the spin, each spin-realization of a charge state is equally probable. We can therefore define $P_{0}=\left\langle 0\left|\rho_{\mathrm{st}}\right| 0\right\rangle, P_{\mathrm{r}^{\prime}}^{\mathrm{r}}=\sum_{\sigma}\left\langle\mathrm{r} \sigma\left|\rho_{\mathrm{st}}\right| \mathrm{r}^{\prime} \sigma\right\rangle$, and $P_{2}=\sum_{\sigma, \sigma^{\prime}}\left\langle\operatorname{L} \sigma \mathrm{R} \sigma^{\prime}\left|\rho_{\text {st }}\right| \operatorname{L} \sigma \mathrm{R} \sigma^{\prime}\right\rangle$. Furthermore the stationary density matrix is diagonal in spin and particle number. Thus the reduced density matrix $\boldsymbol{\rho}$ describing the double dot is given by the $4 \times 4$ matrix

$$
\boldsymbol{\rho}_{\mathrm{st}}=\left(\begin{array}{cccc}
P_{0} & 0 & 0 & 0 \\
0 & P_{\mathrm{L}}^{\mathrm{L}} & P_{\mathrm{R}}^{\mathrm{L}} & 0 \\
0 & P_{\mathrm{L}}^{\mathrm{R}} & P_{\mathrm{R}}^{\mathrm{R}} & 0 \\
0 & 0 & 0 & P_{2}
\end{array}\right) .
$$

The diagonal elements of the density matrix are the probabilities to find the double dot empty $\left(P_{0}\right)$, the left $\left(P_{\mathrm{L}}^{\mathrm{L}}\right)$ or right $\operatorname{dot}\left(P_{\mathrm{R}}^{\mathrm{R}}\right)$ singly occupied, or the two dots simultaneously occupied by one electron $\left(P_{2}\right)$. Superpositions of the two single occupied states are possible $P_{\mathrm{R}}^{\mathrm{L}}=\left(P_{\mathrm{L}}^{\mathrm{R}}\right)^{\star}$.

One can define an effective tensor for $\hat{\Sigma}$, that only depends on the orbital part of the matrix elements (denoted in the following formula by $\chi_{1}, \chi_{2}, \chi_{3}, \chi_{4}$ ) and no longer on the spin variables. The new tensor elements are defined by:

$$
\Sigma_{\chi_{4} \chi_{2}}^{\chi_{3} \chi_{1}}=\sum_{f} \Sigma_{\chi_{4}^{f} \chi_{2}^{i}}^{\chi_{3}^{f} \chi_{1}^{i}}
$$

Here $i$ labels any possible spin-realization for the initial states, $\chi_{1}, \chi_{2}$, and $f$ for the final states $\chi_{3}, \chi_{4}$. (Due to spin degeneracies the two particle states are four fold degenerate, and the left and right states are each two-fold degenerate.) The tunnel tensor $\Sigma_{\chi_{4} \chi_{2}}^{\chi_{3} \chi_{1}}$ is independent of the spin-realization $i$. The spin degeneracy appears only as a prefactor, but does not change the functional form of the elements. For example, $\Sigma_{\mathrm{L}, 0}^{\mathrm{L}, 0}=\sum_{\sigma} \Sigma_{\mathrm{L} \sigma, 0}^{\mathrm{L} \sigma, 0}$ describing the transition from $P_{0}$ to $P_{\mathrm{L}}$ is twice as big for spindegenerate electrons as for spin-less fermions. On the other hand $\Sigma_{\mathrm{L}, \mathrm{L}}^{\mathrm{L}, \mathrm{L}}=\Sigma_{\mathrm{L} \uparrow, \mathrm{L} \uparrow}^{\mathrm{L} \uparrow, \mathrm{L} \uparrow}+\Sigma_{\mathrm{L} \downarrow, \mathrm{L} \uparrow}^{\mathrm{L} \downarrow}=\Sigma_{\mathrm{L} \downarrow, \mathrm{L} \downarrow}^{\mathrm{L} \downarrow, \mathrm{L} \downarrow}+\Sigma_{\mathrm{L} \uparrow, \mathrm{L} \downarrow}^{\mathrm{L} \uparrow, \mathrm{L} \downarrow}$ describing the loss term of $P_{\mathrm{L}}$ is the same for spindegenerate or spin-less fermions since $\Sigma_{\mathrm{L \downarrow}, \mathrm{L} \uparrow}^{\mathrm{L} \downarrow, \mathrm{L} \uparrow}=0=$ $\Sigma_{\mathrm{L} \uparrow, \mathrm{L} \downarrow}^{\mathrm{L} \uparrow, \mathrm{L} \downarrow}$ 
This treatment of the spin allows a general solution of the problem including both, the case of spin polarized electrons and the case of spin degenerate electrons. For the interested reader, we specify the degeneracy of fermions in the further Appendix by the variable $g_{\sigma}$ : $g_{\sigma}=2$ for electrons, $g_{\sigma}=1$ for spin-less fermions.

\section{APPENDIX B: REWRITING KINETIC EQUATION AS BLOCH LIKE EQUATION}

Instead of working with off-diagonal density matrix elements, we can switch to a pseudo spin representation. As any two level system, the $2 \times 2$ hermitian submatrix of the singly occupied states in Eq. (A4) can be treated as $S U(2)$ representation of a pseudo spin Bloch vector $\boldsymbol{I}=\left(P_{\mathrm{R}}^{\mathrm{L}}+P_{\mathrm{L}}^{\mathrm{R}}, i P_{\mathrm{R}}^{\mathrm{L}}-i P_{\mathrm{L}}^{\mathrm{R}}, P_{\mathrm{L}}^{\mathrm{L}}-P_{\mathrm{R}}^{\mathrm{R}}\right)^{T} / 2$. For a complete set of variables, we further introduce $P_{1}=P_{\mathrm{L}}^{\mathrm{L}}+P_{\mathrm{R}}^{\mathrm{R}}$ as the probability of a singly-occupied double dot. Such a pseudo spin representation is often used in the quantum information community. ${ }^{4,5}$ With this change of variables, the dynamics of the double dot system can be mapped on the motion of a spin in an external magnetic field. This is in close analogy to the dynamics of a quantum dot connected to ferromagnetic leads. ${ }^{28,29}$

Due to the serial geometry the external tunneling affects only the z-direction of the pseudo spin and the left and right contacts couple with a different sign to $I_{\mathrm{z}}$. This is captured by the definitions $\hat{\mathbf{n}}_{\mathrm{L}}=(0,0,1)$ and $\hat{\mathbf{n}}_{\mathrm{R}}=(0,0,-1)$, which can be understood as pseudo-spin magnetizations of the leads. With this definitions the occupation probabilities obey the following master equations:

$$
\begin{aligned}
0=\frac{d}{d t} P_{0}= & \sum_{r} \frac{\Gamma_{r}}{\hbar}\left(-g_{\sigma} f_{r}(\bar{E}) P_{0}+\frac{1}{2} f_{r}^{-}(\bar{E}) P_{1}\right)+ \\
& +\sum_{r} \frac{\Gamma_{r}}{\hbar} f_{r}^{-}(\bar{E}) \hat{\mathbf{n}}_{\mathrm{r}} \cdot \mathbf{I} \\
0=\frac{d}{d t} P_{2}= & \sum_{r} \frac{\Gamma_{r}}{\hbar}\left(\frac{g_{\sigma}}{2} f_{r}(\bar{E}+U) P_{1}-f_{r}^{-}(\bar{E}+U) P_{2}\right) \\
& -\sum_{r} \frac{\Gamma_{r}}{\hbar} g_{\sigma} f_{r}(\bar{E}+U) \hat{\mathbf{n}}_{\mathrm{r}} \cdot \mathbf{I} \\
P_{1}= & 1-P_{0}-P_{2}
\end{aligned}
$$

In equilibrium $\left(f_{\mathrm{R}}=f_{\mathrm{L}}\right)$ the diagonal matrix elements are given by the Boltzmann statistics $P_{0}=$ $1 / Z, P_{1}=2 g_{\sigma} \exp \left[-\bar{E} / k_{\mathrm{B}} T\right] / Z, P_{2}=g_{\sigma}^{2} \exp [-(\bar{E}+$ $\left.U) / k_{\mathrm{B}} T\right] / Z, Z=P_{0}+P_{1}+P_{2}$ and the accumulation term as well as all components of the pseudo spin vanish.

The dynamics of the single particle state is described by a Bloch-like equation:

$$
\begin{aligned}
0=\frac{d}{d t} \mathbf{I} & =\left(\frac{d \mathbf{I}}{d t}\right)_{\text {acc. }}-\left(\frac{d \mathbf{I}}{d t}\right)_{\text {rel. }}+\frac{1}{\hbar}(\mathbf{B} \times \mathbf{I}) \\
\left(\frac{d \mathbf{I}}{d t}\right)_{\text {acc. }} & =\sum_{r} \hat{\mathbf{n}}_{\mathrm{r}} \frac{\Gamma_{r}}{2 \hbar}\left[g_{\sigma} f_{r}(\bar{E}) P_{0}+\right. \\
& +\frac{1}{2}\left(g_{\sigma} f_{r}(\bar{E}+U)-f_{r}^{-}(\bar{E})\right) P_{1} \\
& \left.-f_{r}^{-}(\bar{E}+U) P_{2}\right] \\
\left(\frac{d \mathbf{I}}{d t}\right)_{\text {rel. }} & =\frac{1}{2} \sum_{r} \frac{\Gamma_{r}}{\hbar}\left(f_{r}^{-}(\bar{E})+g_{\sigma} f_{r}(\bar{E}+U)\right) \mathbf{I}
\end{aligned}
$$

Three different terms can be identified in the Bloch equation. The term $(d \mathbf{I} / d t)_{\text {acc. }}$ describes the accumulation of pseudo spin in z-direction due to the serial external coupling.

The relaxation-like term $(d \mathbf{I} / d t)_{\text {rel. }}$ limits the amount of pseudo spin. I relaxes isotropic by electrons leaving or entering the singly occupied double dot destroying all pseudo spin components.

The third term looks like a rotation of the pseudo spin around a fictitious magnetic field $\vec{B}=\left(-\Delta, 0, \varepsilon_{\mathrm{ren}}\right)$, where $\varepsilon_{\text {ren }}$ denotes the renormalized level separation

$$
\begin{aligned}
\varepsilon_{\text {ren }}= & \left(E_{\mathrm{L}}-\operatorname{Re}\left[X_{\mathrm{L}}^{(-,-)}(\bar{E})+g_{\sigma} X_{\mathrm{L}}^{(+,-)}(\bar{E}+U)\right.\right. \\
& \left.\left.-\left(g_{\sigma}-1\right) X_{\mathrm{L}}^{(+,-)}\left(\bar{E}+U^{\prime}\right)\right]\right)-(\mathrm{L} \rightarrow \mathrm{R}),
\end{aligned}
$$

where Re denotes the real part. The Cauchy principal value integrals are defined in Eq. (A2).

This third term describes coherent oscillations inside the double dot which mix the accumulated spin in zdirection with the other components. The interdot tunneling characterized by $\Delta$ leads to a precession of the isospin around the $\mathrm{x}$-axes, while the energy separation between the dot levels results in a rotation around the zaxes. It is important to note that the renormalized level separation between the dots changes due to the external coupling and it is not given by the bare level separation $\varepsilon$.

In the following we outline the close analogy between the transport through a serial double dot described here and the dynamics in a spin valve described in Ref. 28,29 . Fig. 7 sketches a spin valve, realized by a single level quantum dot placed between anti-aligned ferromagnets. Relating the pseudo spin $\vec{I}$, in the present work with the real spin $\vec{S}$, in such a spin valve, one can perform the following mapping. The serial setup for the double dot system corresponds to the anti-aligned magnetization of the contacts in the spin valve. Furthermore the interdot tunneling translates to a transverse magnetic field in the single dot, while the level separation $\varepsilon$ corresponds to the magnetic field component along the magnetization of the contacts. Finally the renormalization of the energy levels discussed here was introduced in the spin valve as an exchange field leading to the Hanle effect. ${ }^{29}$ 


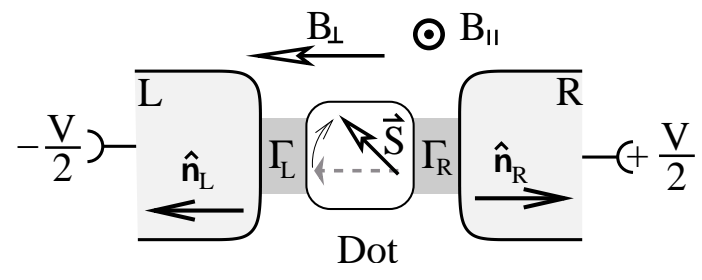

FIG. 7: Sketch of quantum dot spin valve. A single level quantum dot is connected to two ferromagnetic reservoirs with antiparallel magnetization. The spin precesses around an external field with a component transverse $\left(B_{\perp}\right)$ and along $\left(B_{\|}\right)$ the magnetization of the leads. $B_{\|}$is modified by an exchange field arising due to the external coupling. This exchange field is manifest in the transport properties of the spin valve.

According to equation Eq. (6) the stationary current is just given by the $y$-component of the isospin

$$
I=\frac{i e \Delta}{2 \hbar}\left(\rho_{\mathrm{R}}^{\mathrm{L}}-\rho_{\mathrm{L}}^{\mathrm{R}}\right)=\frac{e \Delta}{\hbar} I_{\mathrm{y}}
$$

The system of master equations can be solved analytically and the current as function of bias voltage and gate voltages has the following form:

$$
I \frac{\hbar}{e}=\Delta^{2} \frac{A}{\varepsilon_{\mathrm{ren}}^{2}+B^{2}}
$$

with the factors

$$
\begin{aligned}
A= & \frac{Z_{A}}{N} ; \quad B^{2}=\frac{Z_{0}^{2}}{4}+\frac{\Delta^{2} Z_{B}}{N} \\
Z_{0}= & \left(\sum_{r} \Gamma_{r} f_{r 1}^{-}+g_{\sigma} \Gamma_{r} f_{r 2}\right) \\
N= & g_{\sigma} \sum_{r} \Gamma_{r}\left(f_{r 2}^{-}+g_{\sigma} f_{r 2}\right)\left(f_{r 1}^{-} f_{\bar{r} 1}+g_{\sigma} f_{r 1} f_{\bar{r} 2}\right) \\
& +\sum_{r} \Gamma_{r}\left(f_{r 1}^{-}+g_{\sigma} f_{r 1}\right)\left(f_{r 2}^{-} f_{\bar{r} 1}^{-}+g_{\sigma} f_{r 2} f_{\bar{r} 2}^{-}\right) \\
Z_{A}= & \frac{g_{\sigma} Z_{0}}{4}\left(g_{\sigma}\left(f_{L 2}-f_{R 2}\right)\left(\sum_{r} \Gamma_{r} f_{r 1}\right)\right. \\
& \left.+\left(f_{L 1}-f_{R 1}\right)\left(\sum_{r} \Gamma_{r} f_{r 2}^{-}\right)\right) \\
Z_{B}= & \frac{Z_{0}}{4}\left(\frac{\left(\sum_{r} \Gamma_{r}^{2}\left(f_{r 1}^{-} f_{r 2}^{-}+2 g_{\sigma} f_{r 1} f_{r 2}^{-}+g_{\sigma}^{2} f_{r 1} f_{r 2}\right)\right)}{\Gamma_{L} \Gamma_{R}}\right. \\
& +f_{L 2}^{-}\left(f_{R 1}^{-}+2 g_{\sigma} f_{R 1}\right)+f_{R 2}^{-}\left(f_{L 1}^{-}+2 g_{\sigma} f_{L 1}\right) \\
& \left.+g_{\sigma}^{2}\left(f_{L 1} f_{R 2}+f_{R 1} f_{L 2}\right)\right)
\end{aligned}
$$

Here $\bar{r}$ denotes the opposite of $r$ and we use the abbreviations $f_{r 1}=f_{r}(\bar{E}), f_{r 1}^{-}=1-f_{r 1}, f_{r 2}=f_{r}(\bar{E}+U)$, $f_{r 2}^{-}=1-f_{r 2}$, as well as the approximation $f_{r}\left(E_{L}\right) \approx$ $f_{r}\left(E_{R}\right) \approx f_{r}(\bar{E})$.
* Electronic address: bwunsch@physnet.uni-hamburg.de

1 M. Rontani et al., Phys. Rev. B 69, 85327 (2004).

2 D. Jacob, B. Wunsch, and D. Pfannkuche, Phys. Rev. B 70, 081314(R) (2004); B. Wunsch, D. Jacob, and D. Pfannkuche, Physica E 26, 464 (2005).

3 T. Hayashi, T. Fujisawa, H. D. Cheong, Y. H. Jeong, and Y. Hirayama, Phys. Rev. Lett. 91, 226804 (2003).

${ }^{4}$ T. Fujisawa, T. Hayashi, H.D. Cheong, Y.H. Jeong, and H. Hirayama Physica E 21, 1046 (2004).

${ }^{5}$ V. N. Golovach and D. Loss, Semicond. Sci. Technol. 17 355 (2002). D. Loss and D.P. DiVincenzo, Phys. Rev. A 57120 (1998).

6 W. G. van der Wiel, S. De Franceschi, J. M. Elzerman, T. Fujisawa, S. Tarucha, and L. P. Kouwenhoven, Rev. Mod. Phys. 75, 1 (2003).

7 J. M. Elzerman, R. Hanson, J. S. Greidanus, vanBeveren,L.H.Willems , S. De Franceschi, L. M. K. Vandersypen, S. Tarucha, and L. P. Kouwenhoven, Phys. Rev. B 67,161308(R) (2003); J. R. Petta, A. C. Johnson, C. M. Marcus, M. P. Hanson, and A. C. Gossard, Phys. Rev. Lett. 93, 186802 (2004).

${ }^{8}$ F. R. Waugh, M. J. Berry, C. H. Crouch, C. Livermore, D. J. Mar, R. M. Westervelt, K. L. Campman, and A. C. Gossard, Phys. Rev. B 53, 1413 (1996).

9 L. P Kouwenhoven, D. G. Austing, and S. Tarucha, Rep. Prog. Phys. 64, 701 (2001).

10 A. K. Hüttel, S. Ludwig, K. Eberl, and J. P. Kotthaus, cond-mat/0501012.

11 T. H. Oosterkamp, S. F. Godijn, M. J. Uilenreef, Yu. V. Nazarov, N. C. van der Vaart, and L. P. Kouwenhoven
Phys. Rev. Lett. 80, 4951 (1998).

12 R. H. Blick, D. Pfannkuche, R. J. Haug, K. v. Klitzing, and K. Eberl, Phys. Rev. Lett. 80, 4032 (1998).

13 T. Brandes, physics reports 408, 315 (2005).

14 Yu. V. Nazarov, Physica B 189, 57 (1993).

15 H. Sprekeler, G. Kießlich, A. Wacker, and E. Schöll, Phys. Rev. B 69, 125328 (2004).

16 S.A. Gurvitz Phys. Rev. B 56, 15215 (1997), S.A. Gurvitz Phys. Rev. B 57, 6602 (1998).

17 S.A. Gurvitz and Ya.S. Prager, Phys. Rev. B 53, 15932 (1996).

18 N. C. van der Vaart, S. F. Godijn, Yu. V. Nazarov, C. J. P. M. Harmans, J. E. Mooij, L. W. Molenkamp, and C. T. Foxon, Phys. Rev. Lett. 74, 4702 (1995).

19 T. Ota, K. Ono, M. Stopa, T. Hatano, S. Tarucha, H. Z. Song, Y. Nakata, T. Miyazawa, T. Ohshima, and N. Yokoyama, Phys. Rev. Lett. 93, 066801 (2004).

20 J. Fransson and O. Eriksson, J. Phys.: Condens. Matter 16 L85 (2004); J. Fransson and O. Eriksson, Phys. Rev. B 70, 085301 (2004).

21 J. König, H. Schoeller, and G. Schön, Phys. Rev. Lett. 76, 1715 (1996); J. König, J. Schmid, H. Schoeller, and G. Schön, Phys. Rev. B 54, 16820 (1996); H. Schoeller, in Mesoscopic Electron Transport, edited by L.L. Sohn, L.P. Kouwenhoven, and G. Schön (Kluwer, Dordrecht, 1997); J. König, Quantum Fluctuations in the Single-Electron Transistor (Shaker, Aachen, 1999).

22 U. Hartmann and F. K. Wilhelm, Phys. Rev. B. 67 161307(R) (2003).

23 T. H. Stoof and Yu. V. Nazarov, Phys. Rev. B. 531050 
(1996); B. L. Hazelzet, M. R. Wegewijs, T. H. Stoof, and Yu. V. Nazarov, Phys. Rev. B 63, 165313 (2001).

24 R. Ziegler, C. Bruder, and H. Schoeller, Phys. Rev. B 62, 1961 (2000).

25 M. Tews, Ann. d. Physik 13, 249 (2004).

${ }^{26}$ K. Blum, Density Matrix Theory and Applications, 2nd ed.,(Plenum Press, New York, 1996).

27 H. Schoeller and J. König, Phys. Rev. Lett. 84, 3686 (2000).

28 J. König and J. Martinek, Phys. Rev. Lett. 90, 166602 (2003); M. Braun, J. König, and J. Martinek, Phys. Rev.
B 70, 195345 (2004)

29 M. Braun, J. König, and Jan Martinek, cond-mat/0411477.

${ }^{30}$ K. Ishibashi, M. Suzuki, T. Ida, and Y. Aoyagi, Appl. Phys. Lett. 79, 1864 (2001).

31 D. Pfannkuche, R. H. Blick, R. J. Haug, K. von Klitzing, K. Eberl, Superlattices \& Microstructures 23, 1255 (1998).

32 R. Schleser, T. Ihn, E. Ruh, K. Ensslin, M. Tews, D. Pfannkuche, D. C. Driscoll, and A. C. Gossard, Phys. Rev. Lett. 94206805 (2005). 